Research Paper

\title{
CCR9 Promotes Migration and Invasion of Lung Adenocarcinoma Cancer Stem Cells
}

\author{
Lin $\mathrm{Lu}^{1,2^{*}}$, Huan $\mathrm{Du}^{1 *}$, Haowei Huang1 ${ }^{*}$, Chenxi Wang1, Peipei Wang1, Zhiqiang Zha², Yong Wu1,2, Xia \\ Liu $^{1,2}$, Chengyin Weng ${ }^{1,2}$, Xisheng Fang ${ }^{1,2}$, Baoxiu Li ${ }^{1,2}$, Haibo Mao ${ }^{1,2}$, Lina Wang1,2, Mingmei Guan ${ }^{1,2}{ }^{\varpi}$, \\ Guolong Liu ${ }^{1,2}$ \\ 1. Department of Medical Oncology, Guangzhou First People's Hospital, Guangzhou Medical University, Guangzhou, Guangdong, China, 510180 \\ 2. Department of Medical Oncology, Guangzhou First People's Hospital, School of Medicine, South China University of Technology, Guangzhou, Guangdong, \\ China \\ "These authors contributed equally to this work.
}

$\triangle$ Corresponding authors: Dr. Guolong Liu, Guangzhou First People’s Hospital, Guangzhou Medical University, Guangzhou, Guangdong, China, 510180. Tel: +86-20-81045125, Fax: +86-20-81045125, E-mail: eyglliu@scut.edu.cn or Dr. Mingmei Guan, Tel: +86-20-81045230, Fax: +86-20-81045230, E-mail: 13808815499@163.com

(C) The author(s). This is an open access article distributed under the terms of the Creative Commons Attribution License (https://creativecommons.org/licenses/by/4.0/). See http://ivyspring.com/terms for full terms and conditions.

Received: 2019.10.03; Accepted: 2020.02.22; Published: 2020.03.26

\begin{abstract}
Aim: CC chemokine receptor 9 (CCR9) interacts with its exclusive ligand CCL25, resulting in promoting tumor progression and metastasis. However, the effect and mechanisms of CCR9 on lung adenocarcinoma distant metastasis remain largely unknown. To preliminary clarify the underlying mechanisms, we investigate the correlation between CCR9 and ALDHIA I+cancer stem cells (CSCs), as well as the effect of CCR9 on the migration and invasion of CSCs.

Methods: Immunohistochemistry was performed to detect the expression of CCR9 in lung adenocarcinoma tissues. The correlations of CCR9 with distant metastasis and overall survival were investigated. Serial paraffin-embedded tissue blocks were used to detect ALDHIA1+CSCs expression. The correlations between CCR9 expression and ALDHIAI+CSCs were evaluated. We further studied the effect of CCR9/CCL25 on the migration and invasion of CSCs using transwell assays.

Results: There were positive correlations between CCR9 expression and distant metastasis, as well as poor overall survival. Patients with high CCR9 expression were more likely to develop distant metastasis and demonstrated poorer overall survival than patients with low CCR9 expression. In addition, there was positive correlation between the expression of CCR9 and ALDHIAl in the same tumor microenvironment. ALDHhigh CSCs demonstrated enhanced expression of CCR9 than ALDHlow cells. Further transwell assays demonstrated that the numbers of CSCs migrated or invaded in response to CCL25 were more than that without CCL25 stimulation. Additional application of anti-CCR9 antibody reversed the CCL25-induced migration and invasion of CSCs.

Conclusions: In summary, our study demonstrated that CCR9/CCL25 promoted the migration and invasion of CSCs, which might contribute to distant metastasis and poor overall survival. Our findings provided evidence that CCR9/CCL25 could be used as novel therapeutic targets for lung adenocarcinoma.
\end{abstract}

Key words: CCR9, lung adenocarcinoma, cancer stem cells, migration, invasion

\section{Introduction}

Lung cancer is the most common cancer worldwide, and its morbidity and mortality rank first among all cancers [1]. Non-small cell lung cancer (NSCLC) accounts for about $83 \%$ of lung cancer. As the largest subtype of NSCLC, the incidence of lung adenocarcinoma is increasing yearly [2]. Besides traditional anticancer strategies, the advancements of the targeted therapies and immune checkpoint inhibitors benefit more lung adenocarcinoma patients [3]. However, most of the patients were diagnosed at an advanced stage and with a high possibility to develop distant metastasis. The 5-year survival rate for lung adenocarcinoma remains unsatisfactory [4]. Thus, it's of great importance to find molecular biomarkers 
which could predict metastasis and prognosis.

Chemokine/chemokine receptor axis plays important roles in determining tumor development and metastasis [5-7]. Chemokine receptors were expressed on both immune cells and tumor cells, which could recruit immune cells to the tumor microenvironment, as well as driving metastatic process [6, 7]. CCR9 was identified as an organspecific chemokine receptor, which could specifically bind with thymus-expressed chemokine CCL25/ TECK [8]. The expression of CCR9 and CCL25 have been identified in some solid tumors, including ovarian cancer [9], breast cancer [10], prostate cancer [11], pancreatic cancer [12] and esophageal cancer [13]. Increased CCR9 expression was correlated with poor overall survival $[9,14]$. CCR9/CCL25 axis played an important role in tumor invasion, migration, drug resistance and apoptosis $[15,16]$. The application of anti-CCR9 antibody could inhibit the migration and invasion of tumor cells [17]. Moreover, application of anti-CCR9 monoclonal antibody demonstrated therapeutic efficacy in leukemia cell xenografts. These studies indicated that CCR9/CCL25 demonstrated great potential in tumor-targeted therapy like other chemokine and chemokine receptors $[18,19]$.

CCR9 was also detected highly expressed in nonsmall cell lung cancer (NSCLC) [17]. Increased CCR9 expression was correlated with poor overall survival [14]. However, little is known about the effect and mechanism of CCR9 on distant metastasis in lung adenocarcinoma. In this study, we found that increased expression of CCR9 was correlated with distant metastasis in lung adenocarcinoma patients. There was positive correlation between the expression of CCR9 and ALDH1A1. Expression level of CCR9 on CSCs was much higher than that on non-CSCs, which resulted in promoting the migration and invasion of CSCs.

\section{Material and Methods}

\section{Ethical statement}

This study was approved by the Ethics Committee of Guangzhou First People's Hospital, and written informed consent was obtained from all patients prior to surgery. All procedures involving human participants were in accordance with the ethical standards of the institutional and/or national research committee and with the 1964 Helsinki declaration and its later amendments or comparable ethical standards.

\section{Patients and specimens}

Paraffin-embedded tumor tissues were obtained from 76 cases of lung adenocarcinoma patients who underwent surgery in Guangzhou First People's Hospital between 2007 and 2016. None of the patients had received any anticancer strategies prior to the surgery. All patients were histologically confirmed as lung adenocarcinoma. There were 46 males and 30 females with the average age of 61 years (range from 40 to 78). According to the 8th edition of the International Association for the Study of Lung Cancer (IASLC) published in 2017, there were 43 cases staged as I-II and 33 cases staged as III-IV. The median follow-up period was 60.4 months (range, 1-101 months).

\section{Culture of tumor cells}

Lung adenocarcinoma cell line A549 was purchased from Cellcook Biotech Co.,Ltd (Guangzhou, China) and cultured in complete medium consisting of RMPI 1640 supplemented with $10 \%$ heat-inactivated fetal bovine serum, $100 \mu \mathrm{g} / \mathrm{mL}$ streptomycin, $100 \mathrm{U} / \mathrm{mL}$ penicillin.

\section{Immunohistochemistry}

The paraffin-embedded sections were cut at a thickness of $2 \mu \mathrm{m}$. The immunohistochemical analysis was performed as previously described [20]. After being baked at $60^{\circ} \mathrm{C}$ for 60 minutes, the sections were dewaxed and rehydrated with xylene and graded alcohol. After antigen retrieval, the sections were incubated overnight at $4^{\circ} \mathrm{C}$ with primary antibodies as follows: rabbit anti-CCR9 polyclonal antibody (1:200, Abcam), rabbit anti-CCL25 polyclonal antibody (1:200, ABclonal and 1:200, Signalway Antibody), rabbit anti-ALDH1A1 polyclonal antibody (1:400,Abcam). The sections were washed with PBS and developed with diaminobenzidine (DAB). Finally, the sections were counterstained with hematoxylin, dehydrated and then sealed.

\section{Evaluation of immunohistochemical staining}

Five random non-crossover fields were selected under a microscope at 400 times magnification. The average positive percentage of cells in each field was taken as the percentage of positive cells in the section. And the percent scores were defined as follows: $0, \leq$ $5 \%$; $1,5-25 \% ; 2,25-50 \% ; 3,>50 \%$. The staining intensity scores were defined as follows: 0 , no staining; 1 , weak staining; 2 , moderate staining; 3 , strong staining. The percentage of positive cells and the staining intensity score were multiplied to obtain a final score. The patients were divided into two groups: low expression group ( 0 - 4 points) and high expression group (6-9 points) as previously described [21].

\section{ALDEFLUOR assay}

The ALDEFLUOR has been used as a unique marker to isolate cancer stem cells (CSCs) from lung 
cancer [22]. The ALDEFLUOR ${ }^{+} / \mathrm{ALDH}^{\text {high }}$ subpopulation was isolated using ALDEFLUOR kit (Stem Cell Technologies) as previously described [22, 23]. Tumor cells stained with ALDH inhibitor diethylaminobenzaldehyde (DEAB) was used as negative control.

\section{Flow cytometry}

$\mathrm{ALDH}^{\text {high }}$ or $\mathrm{ALDH}^{\text {low }}$ cells were sorted using flow cytometry. Both ALDH high and ALDH ${ }^{\text {low }}$ cells were incubated with PE-conjugated CCR9 antibody. The expression of CCR9 was detected using flow cytometry as previously described [23].

\section{RNA extraction and real time quantitative PCR (RT-qPCR)}

Total RNA from tumor cells was extracted using TRIzol solution and the cDNA was synthesized as previously described [24, 25]. The resulting cDNA was performed to RT-qPCR to evaluate the relative mRNA levels of CCR9 and internal control GAPDH using the following premiers: 5'- CGATGAACACGA GCCAGTACAAG-3' (F) and 5' - TGCATCTGACTGA CCCACCA-3' (R) for CCR9; 5'-CTCCTCCTGTTCGA CAGTCAGC-3' (F) and 5'-CCCAATACGACCAAAT CCGTT-3' for GAPDH. The relative mRNA values were calculated using the comparative threshold cycle $(2-\Delta \Lambda \mathrm{CT})$ method. And the relative expression levels of CCR9 were normalized to the value of GAPDH. The experiments were repeated at least three times.

\section{Cell migration and invasion}

Cell migration and invasion assays were

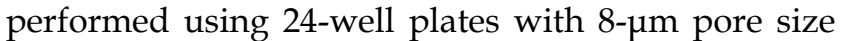
polycarbonate membrane inserts (Corning, USA). For cell invasion assay, polycarbonate membrane inserts were coated with a thin layer of $0.5 \mathrm{mg} / \mathrm{mL}$ Matrigel Basement Membrane Matrix. The lower chambers were filled with $500 \mu \mathrm{L}$ of complete medium. For some experimental group, CCL25 $(100 \mathrm{ng} / \mathrm{ml})$ was added to the lower chamber. For some experimental group, anti-CCR9 $(1 \mu \mathrm{g} / \mathrm{ml}, \mathrm{R} \& \mathrm{D})$ was added to the top chamber. After the cells migrated or invaded to the lower surface, the membrane was stained using crystal violet. The results were calculated by counting the stained cells under an inverted microscope (10 fields per membrane). Each experiment was repeated at least 3 times.

\section{Statistics}

All statistical calculations were performed using software (version 25.0; SPSS Inc., Chicago, IL, USA) or GraphPad Prism 7 (GraphPad software). Student's $\mathrm{t}$-test was used to value the differences between two experiment groups. Chi-square test was used to determine the relationship between CCR9 expression or ALDH1A1 expression with metastasis, and the correlation between CCR9 and ALDH1A1. Survival analysis was performed by the Kaplan-Meier method and log-rank test. Prognostic value of various factors was analyzed by univariate and multivariate analysis (Cox proportional hazards regression model). A two-tailed $P$ value $<0.05$ was considered statistically significant.

\section{Results}

\section{Increased CCR9 expression correlated with distant metastasis and poor outcomes of lung adenocarcinoma patients}

The expression of CCR9 and its ligand CCL25 in the tumor tissues of lung adenocarcinoma patients was investigated using immunohistochemistry. High expression of CCR9 was detected in 48 cases, while 28 cases displayed low CCR9 expression. The representative photomicrographs are shown in Figure 1A and B. However, CCL25 expression was barely detected in lung adenocarcinoma tissues (supplementary Figure 1). We further analyzed the relationship between CCR9 and distant metastasis. As shown in Table 1, there was significant correlation between CCR9 expression and distant metastasis $(P=0.026)$. The high expression rates of CCR9 in patients staged as M0 and M1 were $53.8 \%$ and $83.3 \%$, respectively, while the low expression rates were $46.3 \%$ and $16.7 \%$, respectively.

Table 1. The relationship between CCR9 expression and distant metastasis in lung adenocarcinoma

\begin{tabular}{llllll}
\hline $\begin{array}{l}\text { Distant } \\
\text { metastasis }\end{array}$ & $\mathbf{n}$ & $\begin{array}{l}\text { CCR9 } \\
\mathbf{X}^{2}\end{array}$ & $\boldsymbol{P}$-value \\
\cline { 3 - 4 } & & High expression & Low expression & & \\
\hline M0 & 52 & $28(53.8 \%)$ & $24(46.2 \%)$ & 4.934 & $0.026^{*}$ \\
M1 & 24 & $20(83.3 \%)$ & $4(16.7 \%)$ & & \\
\hline
\end{tabular}

Kaplan-Meier survival analysis was performed to investigate the association between CCR9 expression and the overall survival (OS) of lung adenocarcinoma patients. As shown in Figure 1C, there was significant correlation between CCR9 expression and the OS (Figure $1 C, P=0.007$ ). Patients with high CCR9 expression had poorer OS than those with low CCR9 expression.

\section{Increased expression of ALDHIA1+ cancer stem cells (CSCs) was correlated with distant metastasis and poor overall survival}

Furthermore, the expression of ALDH1A1+ CSCs in the same tumor microenvironment was detected using serial paraffin-embedded sections. ALDH1A1 was highly expressed in 45 cases and lowly expressed in 31 cases. Figure 1D and E showed representative images of ALDH1A1+CSCs. KaplanMeier survival analysis showed that ALDH1A1 
expression could predict the overall survival (OS). Lung adenocarcinoma patients with high ALDH1A1 expression had poorer OS than those with low ALDH1A1 expression $(P=0.003$, Figure $1 F)$. There was positive correlation between the expression of ALDH1A1 ${ }^{+} \mathrm{CSC}$ s and distant metastasis (Table 2, $P=$ 0.016). Patients in the ALDH1A1 high expression group were more likely to develop distant metastasis.
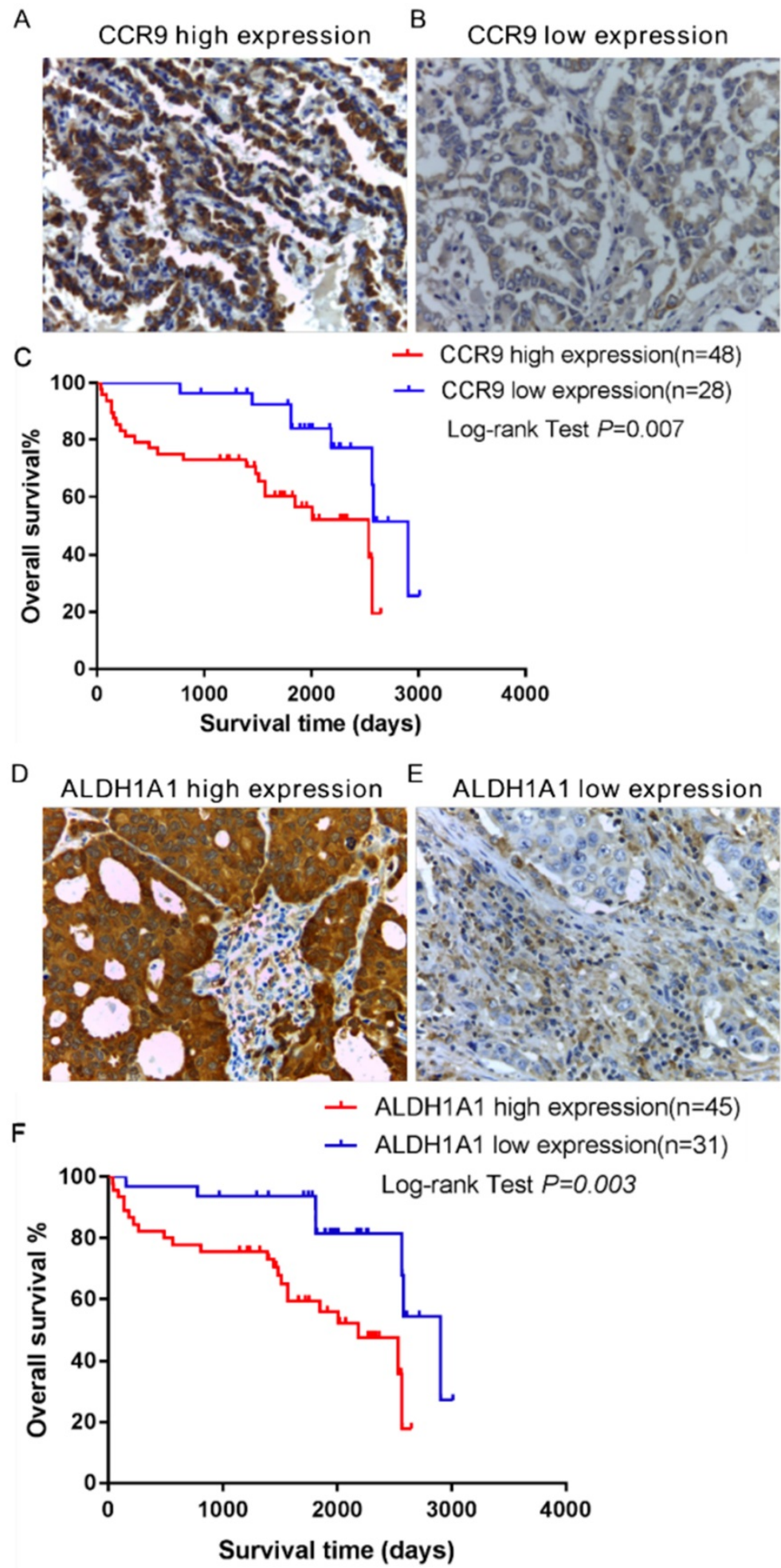

Figure 1. The expression and prognostic value of CCR9 and ALDH1A1 in lung adenocarcinoma patients. Representative microphotographs of CCR9 expression: (A) High CCR9 expression; (B) Low CCR9 expression. (C) Kaplan-Meier curve of overall survival predicted by CCR9 expression. Patients with low CCR9 expression demonstrated OS than patients with high CCR9 expression. Representative microphotographs of high ALDHIAl expression (D) and low ALDHIAl expression (E). (F) Increased ALDHIA1 expression was positively correlated with poor overall survival. Original magnification: 400x. 
Table 2. The relationship between ALDHIA1+CSCs expression and distant metastasis in lung adenocarcinoma

\begin{tabular}{llllll}
\hline $\begin{array}{l}\text { Distant } \\
\text { metastasis }\end{array}$ & $\mathbf{n}$ & \multicolumn{2}{l}{$\begin{array}{l}\text { ALDH1A1 } \\
\mathbf{X}^{2}\end{array}$} & $\boldsymbol{P}$-value \\
\cline { 3 - 4 } & & High expression & Low expression & & \\
\hline M0 & 52 & $26(50.0 \%)$ & $26(50.0 \%)$ & 5.784 & $0.016^{*}$ \\
M1 & 24 & $19(79.2 \%)$ & $5(20.8 \%)$ & & \\
\hline
\end{tabular}

\section{CCR9 expression was positively correlated} with ALDHIAl expression

We further studied the correlation between the expression of CCR9 and ALDH1A. CCR9 and ALDH1A1 were both highly expressed in 42 cases and both lowly expressed in 25 cases. Figure $2 \mathrm{~A}$ and B showed the representative microphotographs from the same patients (high expression and low expression). There was positive correlation between CCR9 expression and ALDH1A1 expression, with a correlation coefficient of $0.602(P<0.001$, Figure $2 C)$. As shown in Figure 2C, the immunostaining scores of
CCR9 in ALDH1A1 high expression group was generally higher than that of in ALDH1A1 low expression group.

\section{CCR9 was highly expressed on ALDH ${ }^{\text {high }}$ cancer stem cells (CSCs)}

To further clarify the correlation between CCR9 and CSCs, we detected the expression of CCR9 on CSCs. Both ALDH ${ }^{\text {high }}$ CSCs and ALDH ${ }^{\text {low }}$ non-CSCs were isolated from lung adenocarcinoma cell line A549 using ALDEFLUOR assay. Expression level of CCR9 on ALDH high CSCs was much higher than that on ALDH ${ }^{\text {low }}$ cells. Representative graphs of flow cytometry were demonstrated in Figure 3A and B. The result was further confirmed by real time quantitative PCR. ALDH ${ }^{\text {high }}$ A549 cells expressed more CCR9 than ALDH ${ }^{\text {low }}$ cells (Figure 3C, $P<0.001$ ).

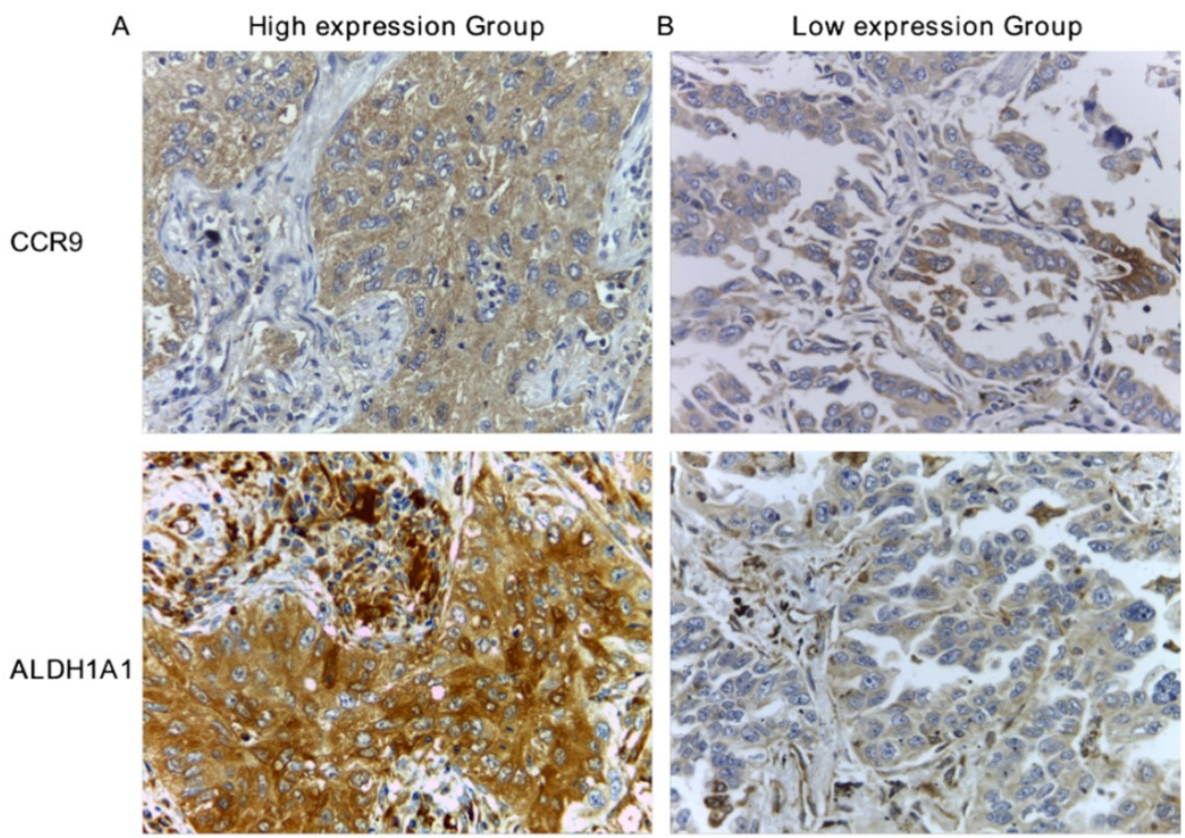

C

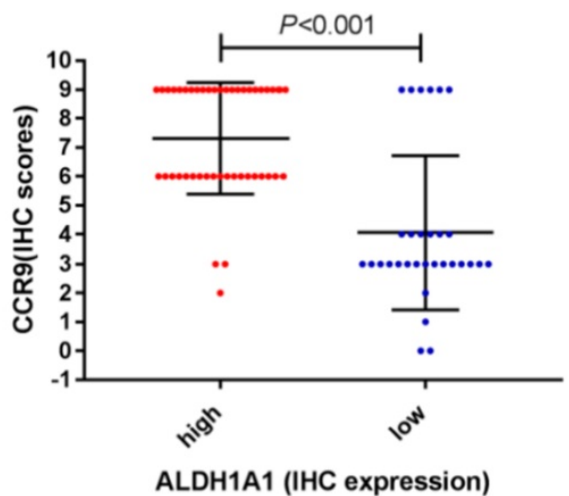

Figure 2. CCR9 expression was positively correlated with ALDHIA1+CSCS expression in lung adenocarcinoma. Representative microphotographs of CCR9 and ALDHIAl expression in the lung adenocarcinoma patients: (A) The same patient with both high CCR9 expression and high ALDH1Al expression; (B) The same patient with both low CCR9 expression and low ALDH1A1 expression. (C) There was a positive correlation between the expression of CCR9 and ALDH1A1+ CSCs. Original magnification: $400 \times$ 

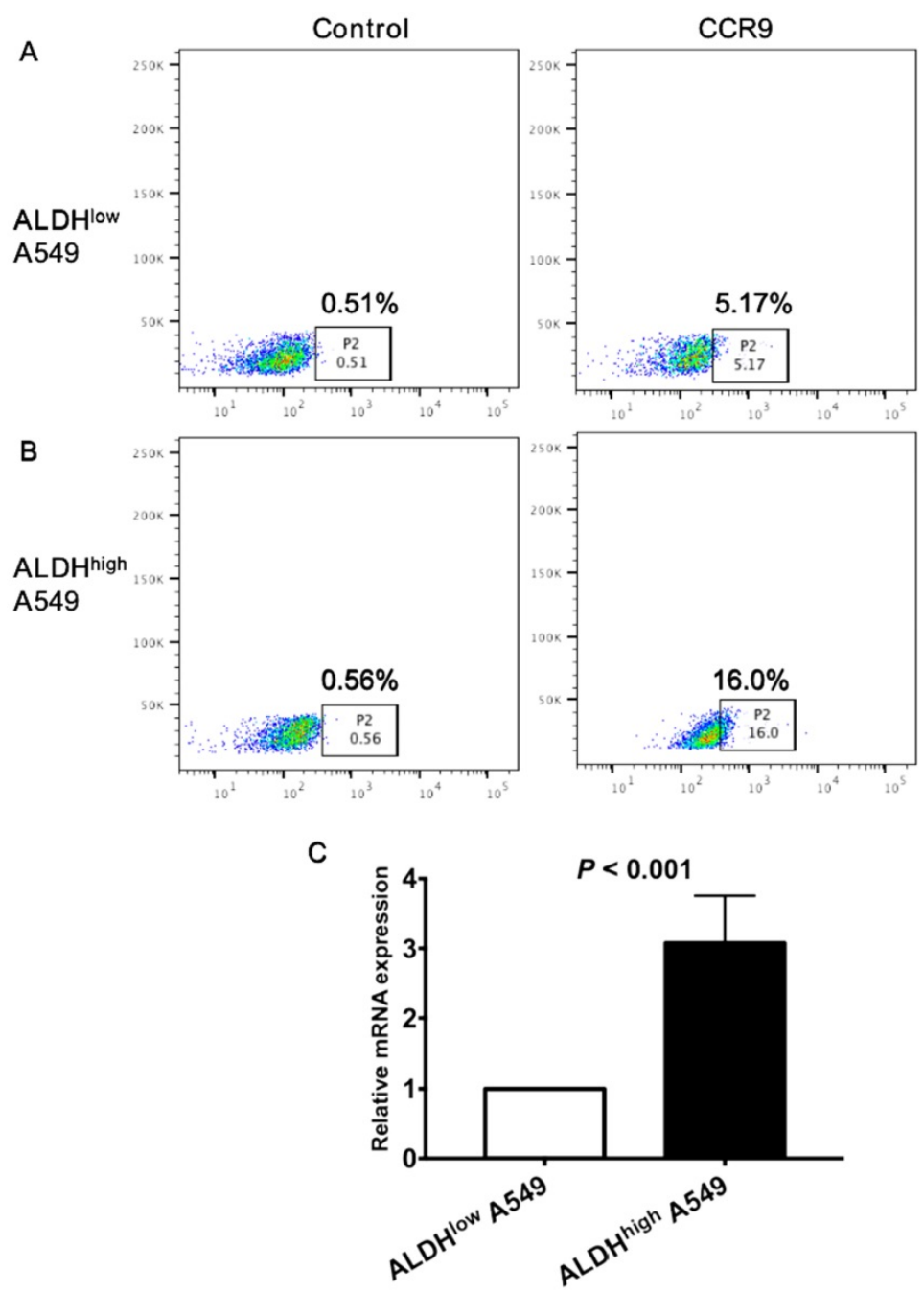

Figure 3. The expression CCR9 on ALDH high CSCs was much higher than that on ALDHlow tumor cells. The expression of CCR9 on ALDH high CSCs (A) and ALDH low non- CSCs (B) was detected using flow cytometry. (C) The expression of CCR9 on ALDHlow cells and ALDHhigh cells were analyzed using real time quantitative PCR.

\section{CCR9/CCL25 promoted the migration and invasion of lung adenocarcinoma cancer stem cells (CSCs)}

To further verify the effect of CCR9/CCL25 on CSCs, we firstly isolated ALDH ${ }^{\text {high }}$ CSCs using flow cytometry as previously described [22, 23]. The numbers of A549 cells migrated in response to CCL25 were much more than the untreated tumor cells (Figure 4A, B). CCL25-induced cell migration was inhibited by additional application of anti-CCR9 antibody (Figure 4A, B). Consistent with the migration assay, there were much more tumor cells invaded to the lower chamber in response to CCL25 stimulation (Figure 4C, D). And the invasive capability of tumor cells in response to CCL25 was inhibited by CCR9 neutralization (Figure 4C, D).

\section{Discussion}

Chemokines/chemokine receptors take part in all stages of tumor development, including immune cells recruitment, neovascularization, tumor proliferation, invasion, and metastasis [26, 27]. CCR9 interacts with its exclusive ligand CCL25 and results in trafficking of both lymphocytes and tumor cells to participate in tumor immunity and tumor development [11, 16, 17, 27-30]. More and more studies have indicated that CCR9 could be viewed as an oncogenic biomarker in predicting metastasis and prognosis for cancer patients. Increased expression of CCR9 and CCL25 have been identified in various tumors, such as nasopharyngeal carcinoma and ovarian cancer $[9,31]$. Upregulated expression of CCR9 and CCL25 were correlated with advanced tumor stage and poor overall survival [9, 31]. Consistantly, our study also demonstrated that increased CCR9 expression was detected in lung adenocarcinoma tissues. High expression of CCR9 was correlated with distant metastasis and poor overall survival in lung adenocarcinoma patients. However, the expression of CCL25 was barely detect in lung adenocarcinoma tissues. Of note, Gupta et al 
failed to detect the expression of CCL25 in lung cancer cells. Instead, they detected the higher serum concentration of CCL25 in lung adenocarcinoma patients than that in the lung squamous cell carcinoma patients and healthy controls [17].

Prostate cancer cells highly expressing CCR9 could promote tumor cell migration and invasion [11]. Expression of CCR9 mediated melanoma specific metastasis to the small intestine [30, 32]. The expression of CCR9 also reduced the sensitivity of tumor cells to chemotherapy, which promoted cancer cells invasion and metastasis [29]. Chen et al engineered colorectal cancer cells to express CCR9, resulting in the promotion of distant metastasis [33]. It's generally accepted that the existence of cancer stem cells (CSCs) in the tumor residue contributing to chemo and radio-resistance, tumor invasion, and metastasis [34, 35]. CCR9 was correlated with chemo-resistance, tumor invasion and metastasis, which indicating that there might be cross-talk between CCR9 and cancer stem cells in the tumor microenvironment.

CCR9/CCL25 has been reported to play important role in attracting stem cells [36]. However, there were little studies concerning the effect of CCR9/CCL25 signal on cancer stem cells. CCR9 was detected on circulating tumor cells of melanoma patients, along with tumor cell migration and distant metastasis [37]. Zhang et al found that CCL25 stimulation up-regulated the expression of epithelial- mesenchymal transition (EMT) markers in breast cancer cells and hepatocellular carcinoma cells[38]. Chen et al detected the expression of CCR9 in colon-cancer-initiating cell lines generated from the early stage of colorectal cancer, which led to the formation of xenograft tumors[39]. Thus, we further investigated the correlation between CCR9 and CSCs. The immunohistochemistry using serial paraffinembedded slides demonstrated that increased expression of ALDH1A1+CSCs was associated with distant metastasis and poor prognosis of lung adenocarcinoma patients. Besides, the expression of CCR9 was positively correlated with ALDH1A1 expression in the same tumor microenvironment. The expression of CCR9 was enhanced on ALDH high CSCs. Additional supplement of CCL25 promoted the migration and invasion of ALDH ${ }^{\text {high }}$ CSCs, which was reversed by the application of CCR9 neutralizing antibody.

In conclusion, our study found that there was positive correlation between CCR9 and ALDH1A1+ CSCs. Increased expression of CCR9 was detected on CSCs, which resulted in the migration and invasion of cancer stem cells through CCR9/CCL25 axis. This might contribute to the distant metastasis of lung adenocarcinoma. This study will provide more evidence for us to further study the application of CCR9 as an effective and novel strategy in inhibiting the metastasis of lung adenocarcinoma.
A

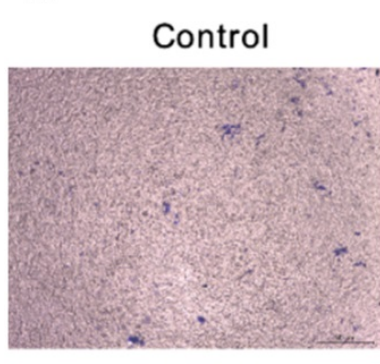

C
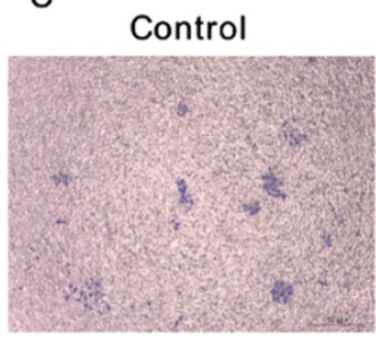

CCL25

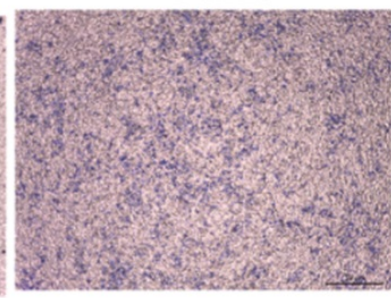

CCL25

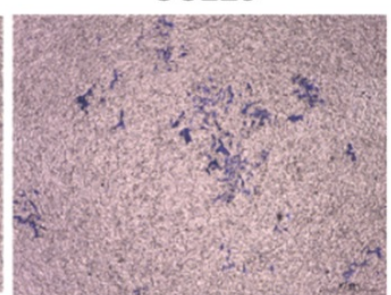

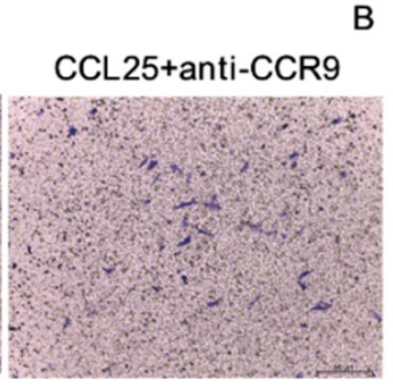
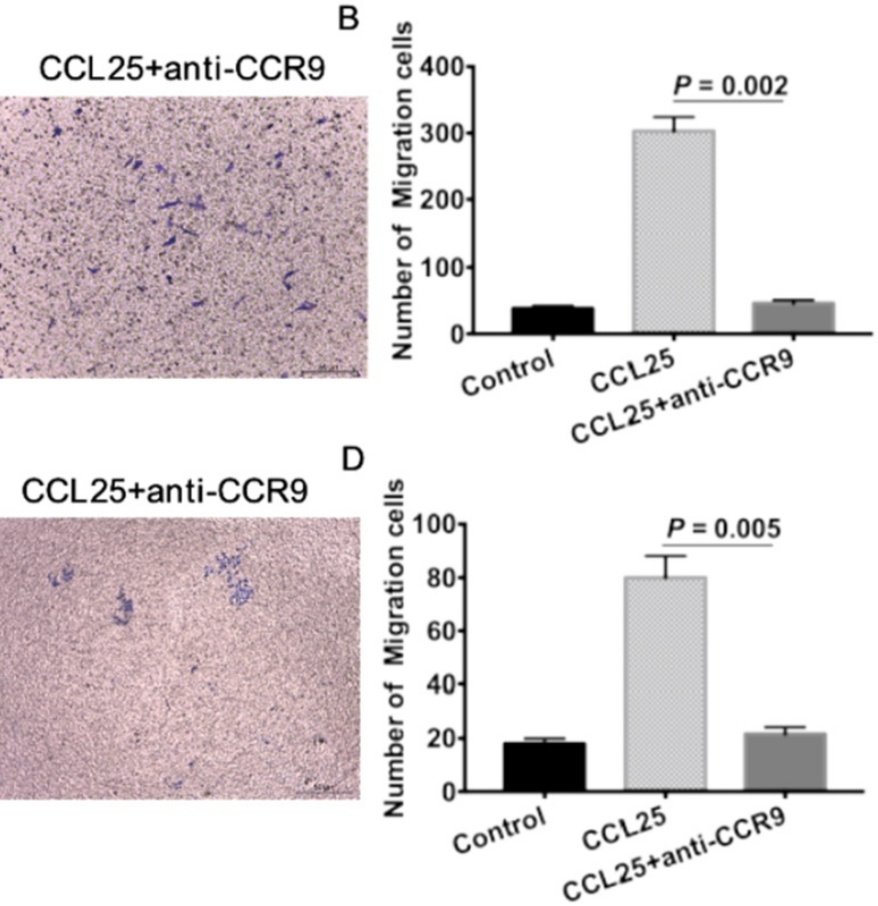

Figure 4. CCR9/CCL25 promoted migration and invasion of ALDH $^{+}$cancer stem cells. ALDHhigh CSCs were isolated using ALDEFLUOR kit. CSCs showed increased migration (A) and invasion (B) capabilities towards CCL25 stimulation. Additional application of CCR9 antibody inhibited CCL25-mediated cell migration (A) and invasion (B). Representative images are shown on the left, and the quantification of the results are shown on the right. Original magnification: $40 \times$. 


\section{Abbreviations}

CCR9: CC chemokine receptor 9; ALDH1A1: aldehyde dehydrogenase 1 family member A1; CSCs: cancer stem cells; NSCLC: Non-small cell lung cancer; RT-qPCR: real time quantitative PCR; GAPDH: glyceraldehyde-3-phosphate dehydrogenase; DEAB: diethylaminobenzaldehyde; OS: overall survival; EMT: epithelial-mesenchymal transition.

\section{Supplementary Material}

Supplementary figure.

http://www.medsci.org/v17p0912s1.pdf

\section{Acknowledgements}

This research was funded by the National Natural Science Foundation of China (No. 81672900 and No. 81502563), the Natural Science Foundation of Guangdong Province, China (No. 2016A030310109), Guangzhou Planned Project of Science and Technology (No. 201904010427 and No.201707010262) and the Fundamental Research Funds for the Central Universities (No. X2yx-D2175050).

\section{Author Contributions}

Conception and design: Lin Lu and Guolong Liu; Laboratory operator: Huan Du, Haowei Huang, Chenxi Wang, and Peipei Wang; Collection and assembly of data: Mingmei Guan and Zhiqiang Zha; Data analysis and interpretation: Yong $\mathrm{Wu}$, Xia Liu and Xisheng Fang; Manuscript writing: Huan Du and Lin Lu; Manuscript revising: Baoxiu Li, Haibo Mao and Lina Wang; Final approval of manuscript: All authors.

\section{Ethics approval and consent to participate}

This study was approved by the Ethics Committee of Guangzhou First People's Hospital, and written informed consent was obtained from all patients prior to surgery. All procedures performed in studies involving human participants were in accordance with the ethical standards of the institutional and/or national research committee and with the 1964 Helsinki declaration and its later amendments or comparable ethical standards.

\section{Availability of data and material}

All data generated or analyzed during this study are included within the article. The datasets used and analyzed during the current study are available from the corresponding author on reasonable request.

\section{Competing Interests}

The authors have declared that no competing interest exists.

\section{References}

1. Bray F, Ferlay J, Soerjomataram I, Siegel RL, Torre LA, Jemal A. Global cancer statistics 2018: GLOBOCAN estimates of incidence and mortality worldwide for 36 cancers in 185 countries. CA: a cancer journal for clinicians. 2018; 68: 394-424

2. Barta JA, Powell CA, Wisnivesky JP. Global Epidemiology of Lung Cancer. Annals of global health. 2019; 85.

3. Chalela R, Curull V, Enriquez C, Pijuan L, Bellosillo B, Gea J. Lung adenocarcinoma: from molecular basis to genome-guided therapy and immunotherapy. Journal of thoracic disease. 2017; 9: 2142-58.

4. Miller KD, Siegel RL, Lin CC, Mariotto AB, Kramer JL, Rowland JH, et al. Cancer treatment and survivorship statistics, 2016. CA: a cancer journal for clinicians. 2016; 66: 271-89.

5. Marcuzzi E, Angioni R, Molon B, Cali B. Chemokines and Chemokine Receptors: Orchestrating Tumor Metastasization. International journal of molecular sciences. 2018; 20.

6. Zhao Q, Zhang P, Qin G, Ren F, Zheng Y, Qiao Y, et al. Role of CXCR7 as a Common Predictor for Prognosis in Solid Tumors: a Meta-Analysis. Journal of Cancer. 2018; 9: 3138-48.

7. Zhou Z, Xia GK, Xiang Z, Liu M, Wei ZW, Yan J, et al. A C-X-C chemokine receptor type 2-dominated crosstalk between tumor cells and macrophages drives gastric cancer metastasis. Clinical cancer research : an official journal of the American Association for Cancer Research. 2019.

8. Youn BS, Kim CH, Smith FO, Broxmeyer HE. TECK, an efficacious chemoattractant for human thymocytes, uses GPR-9-6/CCR9 as a specific receptor. Blood. 1999; 94: 2533-6.

9. Singh R, Stockard CR, Grizzle WE, Lillard JW, Jr., Singh S. Expression and histopathological correlation of CCR9 and CCL25 in ovarian cancer. International journal of oncology. 2011; 39: 373-81.

10. Johnson-Holiday C, Singh R, Johnson E, Singh S, Stockard CR, Grizzle WE, et al. CCL25 mediates migration, invasion and matrix metalloproteinase expression by breast cancer cells in a CCR9-dependent fashion. International journal of oncology. 2011; 38: 1279-85.

11. Singh S, Singh UP, Stiles JK, Grizzle WE, Lillard JW, Jr. Expression and functional role of CCR9 in prostate cancer cell migration and invasion. Clinical cancer research : an official journal of the American Association for Cancer Research 2004; 10: 8743-50.

12. Heinrich EL, Arrington AK, Ko ME, Luu C, Lee W, Lu J, et al. Paracrine Activation of Chemokine Receptor CCR9 Enhances The Invasiveness of Pancreatic Cancer Cells. Cancer microenvironment : official journal of the International Cancer Microenvironment Society. 2013; 6: 241-5

13. Mishan MA, Heirani-Tabasi A, Mokhberian N, Hassanzade M, Kalalian Moghaddam H, Bahrami AR, et al. Analysis of Chemokine Receptor Gene Expression in Esophageal Cancer Cells Compared with Breast Cancer with Insights into Metastasis. Iranian journal of public health. 2015; 44: 1353-8.

14. Zhong Y, Jiang L, Lin H, Li B, Lan J, Liang S, et al. Expression of CC chemokine receptor 9 predicts poor prognosis in patients with lung adenocarcinoma. Diagnostic pathology. 2015; 10: 101

15. Wang C, Liu Z, Xu Z, Wu X, Zhang D, Zhang Z, et al. The role of chemokine receptor $9 /$ chemokine ligand 25 signaling: From immune cells to cancer cells. Oncology letters. 2018; 16: 2071-7

16. Tu Z, Xiao R, Xiong J, Tembo KM, Deng X, Xiong M, et al. CCR9 in cancer: oncogenic role and therapeutic targeting. Journal of hematology \& oncology. 2016; 9: 10.

17. Gupta P, Sharma PK, Mir H, Singh R, Singh N, Kloecker GH, et al. CCR9/CCL25 expression in non-small cell lung cancer correlates with aggressive disease and mediates key steps of metastasis. Oncotarget. 2014; 5: 10170-9.

18. Hu Y, Zhang L, Wu R, Han R, Jia Y, Jiang Z, et al. Specific killing of CCR9 high-expressing acute $\mathrm{T}$ lymphocytic leukemia cells by CCL 25 fused with PE38 toxin. Leukemia research. 2011; 35: 1254-60.

19. Chamorro S, Vela M, Franco-Villanueva A, Carramolino L, Gutierrez J, Gomez $\mathrm{L}$, et al. Antitumor effects of a monoclonal antibody to human CCR9 in leukemia cell xenografts. mAbs. 2014; 6: 1000-12.

20. Lu L, Pan K, Zheng HX, Li JJ, Qiu HJ, Zhao JJ, et al. IL-17A promotes immune cell recruitment in human esophageal cancers and the infiltrating dendritic cells represent a positive prognostic marker for patient survival. Journal of immunotherapy (Hagerstown, Md : 1997). 2013; 36: 451-8.

21. Wang W, Lv L, Pan K, Zhang Y, Zhao JJ, Chen JG, et al. Reduced expression of transcription factor AP-2alpha is associated with gastric adenocarcinoma prognosis. PloS one. 2011; 6: e24897.

22. Luo H, Zeng C, Fang C, Seeruttun SR, Lv L, Wang W. A new strategy using ALDHhigh-CD8+T cells to inhibit tumorigenesis. PloS one. 2014; 9: e103193.

23. Lu L, Tao $\mathrm{H}$, Chang $\mathrm{AE}, \mathrm{Hu}$ Y, Shu G, Chen $\mathrm{Q}$, et al. Cancer stem cell vaccine inhibits metastases of primary tumors and induces humoral immune responses against cancer stem cells. Oncoimmunology. 2015; 4: e990767.

24. Lu L, Liu O, Wang P, Wu Y, Liu X, Weng C, et al. MicroRNA-148b regulates tumor growth of non-small cell lung cancer through targeting MAPK/JNK pathway. BMC cancer. 2019; 19: 209.

25. Feng XY, Lu L, Wang KF, Zhu BY, Wen XZ, Peng RQ, et al. Low expression of CD80 predicts for poor prognosis in patients with gastric adenocarcinoma. Future oncology (London, England). 2019; 15: 473-83.

26. Balkwill FR. The chemokine system and cancer. The Journal of pathology. 2012; 226: 148-57. 
27. Jacquelot N, Enot DP, Flament C, Vimond N, Blattner C, Pitt JM, et al. Chemokine receptor patterns in lymphocytes mirror metastatic spreading in melanoma. The Journal of clinical investigation. 2016; 126: 921-37.

28. Costa MF, Bornstein VU, Candea AL, Henriques-Pons A, Henriques MG, Penido C. CCL25 induces alpha(4)beta(7) integrin-dependent migration of IL-17(+) gammadelta T lymphocytes during an allergic reaction. European journal of immunology. 2012; 42: 1250-60.

29. Johnson EL, Singh R, Johnson-Holiday CM, Grizzle WE, Partridge EE, Lillard JW, Jr., et al. CCR9 interactions support ovarian cancer cell survival and resistance to cisplatin-induced apoptosis in a PI3K-dependent and FAK-independent fashion. Journal of ovarian research. 2010; 3: 15.

30. Letsch A, Keilholz U, Schadendorf D, Assfalg G, Asemissen AM, Thiel E, et al. Functional CCR9 expression is associated with small intestinal metastasis. The Journal of investigative dermatology. 2004; 122: 685-90.

31. Ye LF, Huang J, Zhang LP, Chen Z. Intracellular expression profile and clinical significance of the CCR9-CCL25 chemokine receptor complex in nasopharyngeal carcinoma. The Journal of laryngology and otology. 2015; 129: 1013-9.

32. Amersi FF, Terando AM, Goto Y, Scolyer RA, Thompson JF, Tran AN, et al. Activation of CCR9/CCL25 in cutaneous melanoma mediates preferential metastasis to the small intestine. Clinical cancer research : an official journal of the American Association for Cancer Research. 2008; 14: 638-45.

33. Chen HJ, Sun J, Huang Z, Hou H, Jr., Arcilla M, Rakhilin N, et al. Comprehensive models of human primary and metastatic colorectal tumors in immunodeficient and immunocompetent mice by chemokine targeting. Nature biotechnology. 2015; 33: 656-60.

34. Badrinath N, Yoo SY. Recent Advances in Cancer Stem Cell-Targeted Immunotherapy. Cancers. 2019; 11.

35. Ning N, Pan Q, Zheng F, Teitz-Tennenbaum S, Egenti M, Yet J, et al. Cancer stem cell vaccination confers significant antitumor immunity. Cancer research. 2012; 72: 1853-64.

36. Spinnen J, Ringe J, Sittinger M. CCL25 chemokine-guided stem cell attraction: an assessment of possible benefits and risks. Regenerative medicine. 2018; 13: 833-44.

37. Fusi A, Liu Z, Kummerlen V, Nonnemacher A, Jeske J, Keilholz U. Expression of chemokine receptors on circulating tumor cells in patients with solid tumors. Journal of translational medicine. 2012; 10: 52.

38. Zhang Z, Sun T, Chen Y, Gong S, Sun X, Zou F, et al. CCL25/CCR9 Signal Promotes Migration and Invasion in Hepatocellular and Breast Cancer Cell Lines. DNA and cell biology. 2016; 35: 348-57.

39. Chen HJ, Edwards R, Tucci S, Bu P, Milsom J, Lee S, et al. Chemokine 25-induced signaling suppresses colon cancer invasion and metastasis. The Journal of clinical investigation. 2012; 122: 3184-96. 